\title{
On A Survey Of the Convergence and Stability of the Finite Difference Solutions of Partial Differential Equations
}

\author{
George .N. Emenogu; Okonlia \\ Department of Mathematics Michael Okpara University of Agriculture Umudike Abia State, Nigeria. \\ Department of Mathematic/Statistics AkanuIbiam Federal Polytechnic UwanaAfrikpo
}

\begin{abstract}
Finite difference solution of partial differential equations must satisfy the requirement of convergence and stability if they are to be reasonably accurate. In this paper, we examined convergence and stability criterion of finite difference scheme for solving a partial differential equation. A case which is possible when the cumulative effect of all rounding errors is negligible. Consequently, we investigated the propagation of these errors as $t$ increases by applying the Fourier series method.
\end{abstract}

\section{Introduction}

Several works has been carried out by many researchers on the stability of solutions of partial differential equation. Von Neumann [1,2] during the world war II developed a scheme using Fourier series methods to investigate the growth of errors arising from the arithmetic operation needed to solve a finite difference equation. This we shall use in the paper to investigate the stability of some explicit scheme for solving partial differential equation.

\section{Basic equation and results}

Let $u(x, t)$ be the analytical solution of the partial differential equation and $W_{p, q}$ the solution of its finite difference approximation. Define the error function

$$
\begin{aligned}
& E_{P, q}=u\left(x_{p}, t_{q}\right)-W_{p, q}, \quad P=0,1 \ldots \ldots, N \\
& q=0,1 \ldots \ldots, M
\end{aligned}
$$

Then $E_{p, q}$ satisfies the same difference equation as $W_{p, q}$

Let $E_{p} P=0,1, \ldots N$ denote the errors at mesh point $\left(x_{p}, 0\right)$ along the initial line $t=0$.

To investigate the propagation of these errors as $t$ increases we need to find a solution of the finite difference equation in $E_{p, q}$ that reduces to $E_{p}$ when $t=0$.

To apply the Fourier series method [3], we first express the errors in terms of finite Fourier series. Recall that the finite Fourier series of a function in the an interval $[-L, L]$ is

$$
f(x)=a_{0}+\sum_{n=1}^{\infty} a_{n} \cos \left(\frac{\mathrm{n} \pi}{l}\right) x+\sum_{n=1}^{\infty} b_{n} \sin \left(\frac{\mathrm{n} \pi}{l}\right) x
$$

The complex exponential form [4] of this series is

$$
f(x)=\sum_{n=1}^{\infty} A_{n} e^{i} \frac{\mathrm{n} \pi}{\ell} x
$$

In the above expansions of $f(x), a_{0}, a_{n}, b_{n}$ and $A_{n}$ are constants which have to be determined.

Expressing $E_{p}$ in terms of complex Fourier series in an interval of length $\ell$, we have

$$
E_{p}=\sum_{n=1}^{\infty} A_{n} e^{i} \frac{\mathrm{n} \pi}{\ell} x_{p} \quad(i=\sqrt{-1})
$$

In the domain $D=\{(x, t) \backslash 0 \leq x \leq \ell, 0 \leq t \leq T\}$, the mesh points $\left(x_{p}, t_{p}\right)$ assume the form $(p h, q k)$, where $h$ and $k$ are the mesh sizes.

Where

$$
\therefore E_{p}=\sum_{n=1}^{\infty} A_{n} e^{i \alpha_{n} p h}
$$

$$
\alpha_{0}=\frac{\mathrm{n} \pi}{\ell}=\frac{\mathrm{n} \pi}{N h}(\text { since } \ell=N h), \quad i=\sqrt{-1}
$$

Let us consider the propagation of the error at just one point $\left(x_{p}, 0\right)$ as $t$ increases, such an error has a form $e^{i \beta p h}$. 
Assuming that the corresponding error at $t>0$ has the form

$e^{i \beta p h} e^{\propto q k}$, where $\propto$ is a complex constant, then we may write

Where $\xi=e^{\propto k}$

$E_{P, q}=e^{i \beta p h} \xi^{2}(2.4)$

It is obvious that this form of $E_{P, q}$ ensures that $E_{P, q}$ reduce to $E_{P}=e^{i \beta p h}$ when $q=0$

From equation (2.4), we deduce that the error will not increase as $t=q k$ increases provided $|\xi| \leq 1$

\section{Example 2.1}

Investigate the stability of the explicit finite differential equation

Where $\lambda=\frac{k}{h^{2}}$

$$
W_{i, j+1}=\lambda W_{i-1, j}+(1-2 \lambda) W_{i, j}+\lambda W_{i+1, j}
$$

Solution

To avoid confusion over notation, let us $W_{p, q}$ for the solution of the given difference scheme, and $E_{P, q}$ for the error, since $E_{P, q}$ satisfies the same difference equations as

$W_{p, q}$, we have

Writing $E_{P, q}=e^{i \beta p h} \xi^{2}$

$$
(p=1, \ldots \ldots, N-1, \quad q=1, \ldots \ldots, m)
$$

Equation (2.5) becomes

Or

$$
\begin{gathered}
e^{i \beta p h} \xi^{q+1}=\left[\lambda e^{i \beta(p-1) h}+(1-2 \lambda) e^{i \beta p h}+\lambda e^{i \beta(p+1) h}\right] \xi^{q} \\
\therefore \xi=\lambda e^{-i \beta h}+(1-2 \lambda)+\lambda e^{i \beta h}
\end{gathered}
$$

For stability,

$$
\begin{aligned}
\xi & =1-2 \lambda[1-\cos (B h)] \\
& =1-2 \lambda\left[2 \sin ^{2}\left(\frac{B h}{2}\right)\right] \\
& =1-4 \lambda \sin ^{2}\left(\frac{B h}{2}\right)
\end{aligned}
$$

$$
\begin{gathered}
|\xi| \leq 1 \\
\Rightarrow \quad\left|1-4 \lambda \sin ^{2}\left(\frac{B h}{2}\right)\right| \leq 1 \\
\therefore-2 \leq-4 \lambda \sin ^{2}\left(\frac{B h}{2}\right)<0 \\
1 \geq 2 \lambda \sin ^{2}\left(\frac{B h}{2}\right) \geq 0
\end{gathered}
$$

i.e.

$$
\begin{aligned}
& 0 \leq 2 \lambda \sin ^{2}\left(\frac{B h}{2}\right) \leq 1 \\
& 0 \leq \lambda \sin ^{2}\left(\frac{B h}{2}\right) \leq \frac{1}{2}
\end{aligned}
$$

Since

We must have,

$$
0 \leq \sin ^{2}\left(\frac{B h}{2}\right) \leq 1
$$

$$
0<\lambda \leq \frac{1}{2}
$$

Example 2.2

The hyperbolic equation $U_{t t}=U_{x x}$ is approximated by the explicit scheme

Investigate its stability?

$$
\frac{W_{p, q+1}-2 W_{p, q}+W_{p, q-1}}{k^{2}}=\frac{W_{p+1, q}-2 W_{p, q}+W_{p-1, q}}{h^{2}}
$$

Solution

Let $E_{P, q}=e^{i \beta p h}$ be the error at $t=0$ and $E_{P, q}=e^{i \beta p h} \xi^{q}$ be the error at any time $t$.

$E_{P, q}=U\left(x_{p}, t_{q}\right)-W_{p, q}$ satisfies the same difference equation as $W$ so we have 


$$
\begin{gathered}
E_{p, q+1}=\sigma^{2} E_{p-1, q}+2\left(1-\sigma^{2}\right) E_{P, q}+\sigma^{2} E_{p+1, q}-E_{p, q-1} \\
e^{i \beta p h} \xi^{q+1}=\left\{\left[\sigma^{2} e^{i \beta(p-1) h}+2\left(1-\sigma^{2}\right) e^{i \beta p h}+\sigma^{2} e^{i \beta(p+1) h}\right] \xi^{q}-e^{i \beta p h} \xi^{q-1}\right\} \\
\therefore \xi=\sigma^{2} e^{-i \beta h}+2\left(1-\sigma^{2}\right)+\sigma^{2} e^{i \beta h}-\xi^{-1} \\
\xi+\xi^{-1}=2+\sigma^{2}\left(e^{i \beta h}+e^{i \beta h}-2\right) \\
=2+2 \sigma^{2}(\cos p h-1) \\
\xi+\xi^{-1}=2-4 \sigma^{2} \sin ^{2}\left(\frac{B h}{2}\right) \\
=2\left[1-2 \sigma^{2} \sin ^{2}\left(\frac{B h}{2}\right)\right] \\
\therefore \xi^{2}-2 A+1=0
\end{gathered}
$$

Where $A=1-2 \sigma^{2} \sin ^{2}\left(\frac{B h}{2}\right)$, and $\sigma=\frac{k}{h}$

Note

$$
\xi=\frac{2 A \pm \sqrt{4 A^{2}-4}}{2}=A \pm \sqrt{A^{2}-1}
$$

If $A<-1, \quad \sqrt{A^{2}-1}>0$

If $A>-1, \quad \sqrt{A^{2}-1}>0$

For stability, $|\xi| \leq 1$

$\therefore|\xi|=\left|A+\sqrt{A^{2}-1}\right| \leq 1$

and $\left|\xi_{2}\right|=\left|A-\sqrt{A^{2}-1}\right| \leq 1$

if $A<-1,\left|\xi_{2}\right|>1$, giving instability and

if $A>1,\left|\xi_{1}\right| \leq 1$ and $\left|\xi_{2}\right| \leq 1$

i.e. for

$$
\begin{array}{cc} 
& \left|1-2 \sigma^{2} \sin ^{2}\left(\frac{B h}{2}\right)\right| \leq 1 \\
& -1 \leq 1-2 \sigma^{2} \sin ^{2}\left(\frac{B h}{2}\right) \leq 1 \\
& -2 \leq-2 \sigma^{2} \sin ^{2}\left(\frac{B h}{2}\right) \leq 0 \\
\Rightarrow \quad & 0 \leq \sigma^{2} \sin ^{2}\left(\frac{B h}{2}\right) \leq 1 \\
\Rightarrow \quad & 0 \leq \sigma \leq 1
\end{array}
$$

Thus, the given explicit scheme is stable for $0 \leq \sigma=\frac{k}{h} \leq 1$ and unstable otherwise

1. Convergence Criterion

If the solution $u(x, t)$ of the parabolic equation

$u_{t}=u_{x x}(0<x<1, \quad 0<t)$

hascontinuous and finite partial derivative of sufficiently high order in $x$ and $t$, and if $\sigma t \leq \frac{(\sigma x)^{2}}{2}$

Then

I. The error $e_{i j}$ due to discretization satisfies the inequality

$$
\begin{aligned}
\left|e_{i j}\right| & \leq T\left\{\frac{k}{2}\left|u_{t t}\right|_{\max }+\frac{h^{2}}{12}\left|u_{x x x x}\right|_{\max }\right\} \\
\text { where } 0 & <t \leq T, \quad h=\sigma x \text { and } x=\sigma t
\end{aligned}
$$

II. The numerical solution converge to the analytical solution at a rate that is $0\left(k+h^{2}\right)$

Proof

Let the analytic and the numerical solution at a point $\left(x_{i}, t_{j}\right)$ in the domain of the parabolic partial differential equation

$$
u_{t}=u_{x x}, \quad 0 \leq x \leq \ell, \quad t>0
$$

be denoted by $u\left(x_{i}, t_{j}\right)$ and $W_{i, j}$, respectively thus the error due to discretization is

The simplest explicit finite difference approximation to the parabolic equation (3.1) is

$$
e_{i, j,}=u\left(x_{1}, t_{j}\right)-W_{i, j}
$$

Where $h=\sigma x$ and $k=\sigma t$

$$
\frac{W_{i, j+1}-W_{i, j}}{k}-\frac{W_{1-1, j}-2 W_{i, j}+W_{1+1, j}}{h^{2}}
$$


Using equation (3.2), we get

and substituting this into equation (3.3), we get

$$
W_{i, j}=u\left(x_{1}, t_{j}\right)-e_{i, j}
$$

$$
\begin{gathered}
\frac{u\left(x_{1}, t_{j+1}\right)-u\left(x_{1}, t_{j}\right)}{k}-\frac{u\left(x_{1-1}, t_{j}\right)-2 u\left(x_{1}, t_{j}\right)+u\left(x_{1+1}, t_{j}\right)}{h^{2}} \\
=\frac{e_{i, j+1}-e_{i, j}}{k}-\frac{e_{i,-1 j}-2 e_{i, j}+e_{i+1, j}}{h^{2}}
\end{gathered}
$$

Taylor's expansion of $u(x, t)$ in the variable $t$ gives

$$
\begin{aligned}
& u(x+h, t) \cong u(x, t)+h u_{x}(x, t)+\frac{h^{2}}{2 !} u_{x x}(\eta, t), \\
& u(x-h, t) \cong u(x, t)+h u_{x}(x, t)+\frac{h^{2}}{2 !} u_{x x}\left(\eta_{2}, t\right),
\end{aligned}
$$$$
\left(x<\eta_{1}<x+h\right)
$$$$
\left(x-1, \eta_{2}<x\right)
$$

i.e. we may write

$$
\begin{gathered}
u_{t}\left(x_{i}, \xi_{j}\right)-u_{x x}\left(\eta_{i}, t_{j}\right)=\frac{1}{k}\left(e_{i, j+1}-e_{i, j}\right)-\frac{1}{h^{2}}\left(e_{i-1, j}-2 e_{i, j}+e_{i+1, j}\right) \\
\therefore \quad e_{i, j+1}=\lambda e_{i-1, j}+(1-2 \lambda) e_{i, j}+\lambda e_{i+1, j}+k\left[u_{t}\left(x_{i}, \xi_{j}\right)-u_{x x}\left(\eta_{i}, t_{j}\right)\right](3.4
\end{gathered}
$$

Where $\lambda=\frac{k}{h^{2}}, \quad \eta_{i}=x_{i}+\propto h, \quad \xi_{j}=t_{j}+\beta k$

$$
(-1<\alpha<1), \quad(0<\beta<k)
$$

Let $\left|u_{t}\left(x_{i}, \xi_{j}\right)-u_{x x}\left(\eta_{i}, t_{j}\right)\right|_{\max }=M$

and

i.e.

$$
\left|e_{i, j}\right| \leq \lambda\left|e_{i-1, j}\right|+|(1-2 \lambda)|\left|e_{i, j}\right|+\lambda\left|e_{i+1, j}\right|+k\left|u_{t}\left(x_{i}, \xi_{j}\right)-u_{x x}\left(\eta_{i}, t_{j}\right)\right|
$$

$$
\begin{gathered}
E_{j+1} \leq\{\lambda+(1-2 \lambda)+\lambda\} E_{j}+k m=E_{j}+k m \\
\therefore \quad E_{j+1} \leq E_{j}+k m
\end{gathered}
$$

i.e.

But $E_{0}=0$ and $t_{j}=j k$

$$
E_{j} \leq E_{j-1}+k m \leq E_{j-2}+2 k m \leq E_{j-3}+3 k m \leq E_{0}+J k m
$$

Since $\left|e_{i, j}\right|=\left|u_{i, j}-W_{i, j}\right| \leq E_{j} \forall j$ it follows that

$$
\therefore \quad E_{j} \leq t_{j} m
$$

As $h \rightarrow 0$ and $k \rightarrow 0$

$$
\begin{gathered}
M \rightarrow\left[u_{t}\left(x_{i}, t_{j}\right)-u_{x x}\left(x_{i}, t_{j}\right)\right]=0 \\
E_{j} \rightarrow 0 \text { as } \sigma x \rightarrow 0 \text { and } \sigma t \rightarrow 0
\end{gathered}
$$

$$
W \rightarrow u \text { as } \sigma x \text { and } \sigma t \text { both tend to zero provided } \lambda \leq \frac{1}{2}
$$

\section{Conclusion}

The above proof shows that, for the finite difference solution to be convergent, $u_{t}$ and $u_{x x}$ must be uniformly continuous and bounded throughout the solution domain.

Besides, in example (2.1) and (2.2), we saw that for the stability of a finite difference scheme for solving P.D.E to be possible, the cumulative effect of all rounding errors must be negligible.

\section{References}

[1]. Smith, G.D; Numerical solution of Partial Differential Equations. Oxford University Press (1965), page 70

[2]. Greenspan. D and Casulli, V.; Numerical Analysis for applied mathematics, Science and Engineering, (1988) Oxford University Press.

[3]. James W. Brown, Ruel V. Churchill: Fourier series and Boundary Value problems. International Students Edition, McGraw-Hill Book company Inc. (1941)

[4] Murray R. Spiegel; Theory and problems of complex variables: Schaum's outline series, McGraw Hill Book company Singapore (1981). 